T. Kubota and M. Yoshida

Nagoya Math. J.

Vol. 163 (2001), 1-11

\title{
A NOTE ON THE CONGRUENT DISTRIBUTION OF THE NUMBER OF PRIME FACTORS OF NATURAL NUMBERS
}

\author{
TOMIO KUBOTA AND MARIKO YOSHIDA
}

Abstract. Let $n=p_{1} p_{2} \cdots p_{r}$ be a product of $r$ prime numbers which are not
necessarily different. We define then an arithmetic function $\mu_{m}(n)$ by
\[ \mu_{m}(n)=\rho^{r} \quad\left(\rho=e^{2 \pi i / m}\right), \]

where $m$ is a natural number. We further define the function $L\left(s, \mu_{m}\right)$ by the Dirichlet series

$$
L\left(s, \mu_{m}\right)=\sum_{n=1}^{\infty} \frac{\mu_{m}(n)}{n^{s}}=\prod_{p}\left(1-\frac{\rho}{p^{s}}\right)^{-1} \quad(\operatorname{Re} s>1),
$$

and will show that $L\left(s, \mu_{m}\right),(m \geq 3)$, has an infinitely many valued analytic continuation into the half plane $\operatorname{Re} s>1 / 2$.

\section{$\S 1$. Introduction}

Let $n=p_{1} p_{2} \cdots p_{r}$ be a product of $r$ prime numbers which are not necessarily different. We define then an arithmetic function $\mu_{m}(n)$ by

$$
\mu_{m}(n)=\rho^{r} \quad\left(\rho=e^{2 \pi i / m}\right)
$$

where $m$ is a natural number. In the case of $m=2, \mu_{2}(n)$ is related to the Möbius function $\mu(n)$ as

$$
\mu(n)=\left\{\begin{aligned}
\mu_{2}(n) & \text { if } p_{1}, p_{2}, \ldots, p_{r} \text { are different } \\
0 & \text { otherwise }
\end{aligned}\right.
$$

We define the function $L\left(s, \mu_{m}\right)$ by the Dirichlet series

$$
L\left(s, \mu_{m}\right)=\sum_{n=1}^{\infty} \frac{\mu_{m}(n)}{n^{s}} \quad(\operatorname{Re} s>1) .
$$

Received January 26, 2000.

2000 Mathematics Subject Classification: Primary: 11N37, Secondary: 11M41. 
On the other hand, we denote by $N_{j}(x)(0 \leq j<m)$, the number of natural numbers $n$ satisfying $\mu_{m}(n)=\rho^{j}$ and $n \leq x$ for a given positive real number $x$. Namely, $N_{j}$ is the number of those natural numbers $n$ not exceeding $x$ which are products of $r$ prime factors such that $r \equiv j(\bmod m)$.

The Dirichlet series $L\left(s, \mu_{m}\right)$ has the Euler product

$$
L\left(s, \mu_{m}\right)=\prod_{p}\left(1-\frac{\rho}{p^{s}}\right)^{-1} .
$$

If $m=2$, we have the equality

$$
\begin{aligned}
L\left(s, \mu_{2}\right) & =\prod_{p}\left(1+\frac{1}{p^{s}}\right)^{-1}=\prod_{p}\left(1-\frac{1}{p^{2 s}}\right)^{-1} \prod_{p}\left(1-\frac{1}{p^{s}}\right) \\
& =\zeta(2 s) \zeta(s)^{-1}
\end{aligned}
$$

with the Riemann zeta function $\zeta(s)$. Accordingly, $L\left(s, \mu_{2}\right)$ is closely related to $\zeta(s)^{-1}$. If, however, $m \geq 3$, then $L\left(s, \mu_{m}\right)$ is of considerably different nature, although a product formula

$$
\begin{aligned}
\prod_{k=1}^{m-1} L\left(s, \mu_{m}^{k}\right) & =\prod_{k=1}^{m-1} \prod_{p}\left(1-\frac{\rho^{k}}{p^{s}}\right)^{-1}=\prod_{p}\left(1-\frac{1}{p^{m s}}\right)^{-1} \prod_{p}\left(1-\frac{1}{p^{s}}\right) \\
& =\zeta(m s) \zeta(s)^{-1}
\end{aligned}
$$

similar to (3) holds, where

$$
L\left(s, \mu_{m}^{k}\right)=\sum_{n=1}^{\infty} \frac{\mu_{m}(n)^{k}}{n^{s}}
$$

The aim of the present paper is to investigate $L\left(s, \mu_{m}\right)$ to some extent, and, as a result, to obtain some informations on $N_{0}, N_{1}, \ldots, N_{m-1}$.

\section{$\S 2$. Analytic continuation of $L\left(s, \mu_{m}\right)$}

In this article, we will show that $L\left(s, \mu_{m}\right),(m \geq 3)$, has an infinitely many valued analytic continuation into the half plane $\operatorname{Re} s>1 / 2$.

TheOrem 1. Assume $\operatorname{Re} s_{0}>1$ and let $C$ be a smooth path starting from $s_{0}$, remaining in the half plane $\operatorname{Re} s>1 / 2$, and not passing 1 or any zero of $\zeta(s)$. Then, $L\left(s, \mu_{m}\right)$ in $(1)$ can be continued analytically along $C$. 
The analytic continuation thus obtained can be expressed as

$$
L\left(s, \mu_{m}\right)=\zeta(s)^{\rho} G(s)
$$

in the half plane $\operatorname{Re} s>1 / 2$, where $G(s)$ is a holomorphic function in the same region.

Proof. For a moment, suppose $\operatorname{Re} s>1$. Then,

$$
\frac{d}{d s} \log \zeta(s)=-\sum_{p} \frac{d}{d s} \log \left(1-\frac{1}{p^{s}}\right)=-\sum_{p} \frac{\frac{1}{p^{s}}}{1-\frac{1}{p^{s}}} \log p=-\sum_{p} \sum_{k=1}^{\infty} \frac{\log p}{p^{k s}}
$$

and by (2)

$$
\begin{aligned}
\frac{d}{d s} \log L\left(s, \mu_{m}\right) & =-\sum_{p} \frac{d}{d s} \log \left(1-\frac{\rho}{p^{s}}\right)=-\sum_{p} \frac{\frac{\rho}{p^{s}}}{1-\frac{\rho}{p^{s}}} \log p \\
& =-\sum_{p} \sum_{k=1}^{\infty} \frac{\rho^{k} \log p}{p^{k s}} .
\end{aligned}
$$

Therefore, if we put

$$
F(s)=\frac{d}{d s} \log L\left(s, \mu_{m}\right)-\rho \frac{d}{d s} \log \zeta(s),
$$

then

$$
F(s)=\sum_{p} \sum_{k=2}^{\infty} \frac{\left(\rho-\rho^{k}\right) \log p}{p^{k s}} .
$$

This series is absolutely convergent in the half plane $\operatorname{Re} s>1 / 2$, because $\left|\rho-\rho^{k}\right| \leq 2$ and

$$
\sum_{p} \sum_{k=2}^{\infty} \frac{\log p}{p^{k \sigma}}=\sum_{p} \frac{\frac{1}{p^{2 \sigma}}}{1-\frac{1}{p^{\sigma}}} \log p<\frac{\sqrt{2}-1}{\sqrt{2}} \sum_{p} \frac{\log p}{p^{2 \sigma}}<\sum_{n=1}^{\infty} \frac{\log n}{n^{2 \sigma}}<\infty
$$

with $\sigma=\operatorname{Re} s>1 / 2$. Thus, $F(s)$ is holomorphic and one-valued in the half plane $\operatorname{Re} s>1 / 2$, and the analytic continuation of $L\left(s, \mu_{m}\right)$ is obtained by

$$
\begin{aligned}
L\left(s, \mu_{m}\right) & =L\left(s_{0}, \mu_{m}\right) \exp \left(\int_{s_{0}}^{s}\left[\rho \frac{d}{d s} \log \zeta(s)+F(s)\right] d s\right) \\
& =\exp \left(\int_{s_{0}}^{s} \rho \frac{d}{d s} \log \zeta(s) d s\right) \cdot L\left(s_{0}, \mu_{m}\right) \cdot \exp \left(\int_{s_{0}}^{s} F(s) d s\right) .
\end{aligned}
$$


Furthermore, since

$$
\exp \left(\int_{s_{0}}^{s} \rho \frac{d}{d s} \log \zeta(s) d s\right)=\frac{\zeta(s)^{\rho}}{\zeta\left(s_{0}\right)^{\rho}}
$$

holds as an equality between many valued functions, we can correspondingly put

$$
G(s)=\zeta\left(s_{0}\right)^{-\rho} L\left(s_{0}, \mu_{m}\right) \cdot \exp \left(\int_{s_{0}}^{s} F(s) d s\right)
$$

where $\zeta\left(s_{0}\right)^{\rho}$ is uniquely determined by the Euler product of $\zeta(s)$ as

$$
\zeta\left(s_{0}\right)^{\rho}=\exp \left(-\rho \sum_{p} \log \left(1-\frac{1}{p^{s_{0}}}\right)\right) .
$$

In these formulas, $s$ is an arbitrary complex number with $\operatorname{Re} s>1 / 2$, the integral is taken along the path $C$, and $s$ is the end of $C$. Hence, we obtain the assertion of the theorem.

COROllary. If $m \geq 3$, then $L\left(s, \mu_{m}\right)$ is many valued, and has a logarithmic singularity like $(s-1)^{-\rho}$ at 1 , and has logarithmic singularities also at zeros of $\zeta(s)$ in the half plane $\operatorname{Re} s>1 / 2$.

Remark 1. The only pole of $\frac{d}{d s} \log \zeta(s)$ is 1 , and the residue is -1 . At a zero of $\zeta(s)$ of order $g, \frac{d}{d s} \log \zeta(s)$ has a pole of order 1 , and the residue is $g$. Accordingly, $\rho \frac{d}{d s} \log \zeta(s)$ has a pole of order 1 at 1 and at zeros of $\zeta(s)$, and the residues are $-\rho$ and $g \rho$, respectively. If $C$ in the Theorem turns around 1 once in the positive direction, then the analytic continuation is multiplied by $e^{-2 \pi i \rho}$. If $C$ turns around a zero of $\zeta(s)$ of order $g$ once in the positive direction, then the analytic continuation is multiplied by $e^{2 \pi i g \rho}$.

If $m=2$, then $-\rho$ and $g \rho$ in the Theorem are rational integers so that $L\left(s, \mu_{2}\right)$ is one-valued.

THEOREM 2. Assume $m \geq 3$, then an asymptotic formula of the form

$$
\sum_{n \leq x} \mu_{m}(n)=o\left(x^{\alpha}\right)
$$

can not hold, whenever $\alpha<1$. 
Proof. Put

$$
S_{m}(\alpha)=\sum_{n \leq x} \mu_{m}(n)
$$

and suppose that (4) is true for an $\alpha<1$. Then,

(5) $\sum_{n=1}^{\infty} \frac{\mu_{m}(n)}{n^{s}}=\sum_{n=1}^{\infty} \frac{S_{m}(n)-S_{m}(n-1)}{n^{s}}=\sum_{n=1}^{\infty} S_{m}(n)\left(\frac{1}{n^{s}}-\frac{1}{(n+1)^{s}}\right)$

and there exists a positive constant $c_{1}$ such that

$$
\left|S_{m}(n)\right|<c_{1} n^{\alpha}
$$

for all $n \geq 1$. Therefore, we have

$$
S_{m}(n)\left(\frac{1}{n^{s}}-\frac{1}{(n+1)^{s}}\right)=S_{m}(n) \frac{1}{s} \int_{n}^{n+1} x^{-s-1} d x
$$

and

$$
\left|S_{m}(n)\left(\frac{1}{n^{s}}-\frac{1}{(n+1)^{s}}\right)\right| \leq c_{1} n^{\alpha} \cdot \frac{1}{s} n^{-s-1}
$$

so that the series in (1) converges at $s=\alpha+\varepsilon$ for every $\varepsilon>0$. It follows from this and from the general theory of Dirichlet series that $L\left(s, \mu_{m}\right)$ is holomorphis and one-valued in the half plane $\operatorname{Re} s>\alpha$, which is a contradiction, because $s=1$ is a logarithmic singularity of $L\left(s, \mu_{m}\right)$ as shown in the Corollary.

$\S 3$. Asymptotic properties of $N_{j}(x),(j=0,1, \ldots, m-1)$

A version of the prime number theorem is

$$
\sum_{n \leq x} \mu(n)=o(x)
$$

and Riemann's hypothesis is equivalent to the fact that

$$
\sum_{n \leq x} \mu(x)=o\left(x^{\alpha}\right)
$$

holds for every $\alpha>1 / 2$.

If $m=2$, the following theorem on the asymptotic behavior of $N_{0}, N_{1}$ is easily deduced from (6) and (7). For the sake of completeness, we state the fact as a theorem with proof. 
TheOREm 3. Let $N_{0}(x)$ and $N_{1}(x)$ be as in $\S 1$ with $m=2$. Then, both $N_{0}(x)$ and $N_{1}(x)$ are $\frac{1}{2} x+o(x)$. If $(7)$ is true, then both $N_{0}(x)$ and $N_{1}(x)$ are $\frac{1}{2} x+o\left(x^{\alpha}\right)$.

Proof. It is enough to treat the second assertion. The definitions of $N_{0}(x), N_{1}(x)$ and $\mu_{2}(x)$ imply

$$
\sum_{n \leq x} \mu_{2}(x)=N_{0}(x)-N_{1}(x)
$$

On the other hand, since

$$
\mu_{2}(n)=\sum_{k^{2} \mid n} \mu\left(\frac{n}{k^{2}}\right)
$$

the left hand side of (7) is equal to

$$
\sum_{n \leq x} \sum_{k^{2} \mid n} \mu\left(\frac{n}{k^{2}}\right)=\sum_{k=1}^{\infty} \sum_{n^{\prime} \leq x / k^{2}} \mu\left(n^{\prime}\right) \quad\left(n=k^{2} n^{\prime}\right) .
$$

So, putting

$$
S(x)=\sum_{n \leq x} \mu(x), \quad S_{2}(x)=\sum_{n \leq x} \mu_{2}(x)
$$

we have

$$
S_{2}(x)=\sum_{k=1}^{\infty} S\left(\frac{x}{k^{2}}\right) .
$$

Under the assumption (7), there is a constant $c_{2}$ such that

$$
|S(x)|<c_{2} x^{\alpha}
$$

for all $x>0$. Hence,

$$
\left|x^{-\alpha} S_{2}(x)\right| \leq \sum_{k=1}^{\infty} x^{-\alpha}\left|S\left(\frac{x}{k^{2}}\right)\right|
$$

and

$$
x^{-\alpha}\left|S\left(\frac{x}{k^{2}}\right)\right| \leq c_{2} x^{-\alpha}\left(\frac{x}{k^{2}}\right)^{\alpha}=c_{2} \frac{1}{k^{2 \alpha}} .
$$


This means that the series in (10) has an absolutely convergent majorant, and, again by the assumption (7), each term $x^{-\alpha}\left|S\left(x / k^{2}\right)\right|$ tends to 0 as $x \rightarrow \infty$. Thus, we have

$$
\lim _{x \rightarrow \infty} x^{\alpha} S_{2}(x)=0
$$

or

$$
N_{0}(x)-N_{1}(x)=o\left(x^{\alpha}\right)
$$

as desired.

Now, it is clear that

$$
N_{0}(x)+N_{1}(x) \sim x
$$

Therefore the Theorem follows from (11) and (12).

Remark 2. Applying Möbius' inversion formula to (9), it is also shown similarly to the above proof that $S_{2}(x)=o\left(x^{\alpha}\right)$ implies $S(x)=o\left(x^{\alpha}\right)$.

We can ask whether similar asymptotic properties as appeared in Theorem 3 exist or not for $N_{j}(x)$ defined in $\S 1$, too. It seems to be fairly hard to answer this kind of question. But, at least, the following theorem is valid:

THEOREM 4. If $m \geq 3$, and if asymptotic formulas of the form

$$
N_{j}(x)=\nu_{j} x+o\left(x^{\alpha}\right) \quad(\alpha<1)
$$

exist for all $j=0,1, \ldots, m-1$, then $\nu_{0}, \nu_{1}, \ldots, \nu_{m-1}$ can not be all equal.

Proof. Assume $\nu_{0}=\nu_{1}=\cdots=\nu_{m-1}$. Then, since

$$
S_{m}(x)=\sum_{j=0}^{m-1} \rho^{j} N_{j}(x),
$$

it turns out that

$$
S_{m}(x)=o\left(x^{\alpha}\right)
$$

which contradicts Theorem 2 . 


\section{$\S 4$. A computational experiment}

Theorem 4 denies the possibility to get an asymptotic formula $N_{j}(x)=$ $\frac{1}{m} x+o\left(x^{\alpha}\right)(j=0,1, \ldots, m-1)$ for $\alpha<1$. But, for $\alpha=1$, the possibility still remains. While, in this direction, the authors still do not possess any theoretical results, they made an experiment by Mathematica in order to examine the behavior of $\frac{1}{x} N_{j}(x)$ as $x \rightarrow \infty$ in the case of $m=3$. The computation up to $x=3 \times 10^{8}$ made it plausible that $\frac{1}{x} N_{j}(x),(j=0,1,2)$, tend to a common limit $1 / 3$.

From Theorem 3, we see that, roughly speaking, the distribution of the number of prime factors of natural numbers is uniform modulo 2 . Theorem 4 shows, however, the distribution is not uniform modulo $m,(m \geq 3)$, if the uniformity is defined rather in a strong sense that $\alpha<1$. Nevertheless, the above computational data allude that the distribution in question is uniform in the weaker sense with $\alpha=1$.

Computational investigation supplies some more facts. Fig. 1 shows the behavior of

$$
\frac{1}{\sqrt[3]{x}} \sum_{n \leq x} \mu_{3}(n) .
$$

up to $3 \times 10^{8}$ plotted at every $10^{4}$ of $n$. While

$$
\frac{1}{x} \sum_{n \leq x} \mu_{3}(n)
$$

probably tends to 0 as $x \rightarrow \infty$, the product of (14) and $x^{3 / 2}$ draws the curve in Fig. 1. So, Fig. 1 shows the behavior of (14) magnified by the factor $x^{3 / 2}$. It is remarkable that the curve in Fig. 1 is fairly smooth.

Fig. 2 shows the behavior of

$$
\frac{1}{\sqrt{x}} \sum_{n \leq x} \mu_{2}(n)
$$

up to $3 \times 10^{8}$ plotted at every $10^{4}$ of $n$. The result is far more disorderly than Fig. 1.

Tables 1 and 2 show actual values of (13) and (15) restricting $n$ to multiples of $10^{7}$. 
Figure 1:

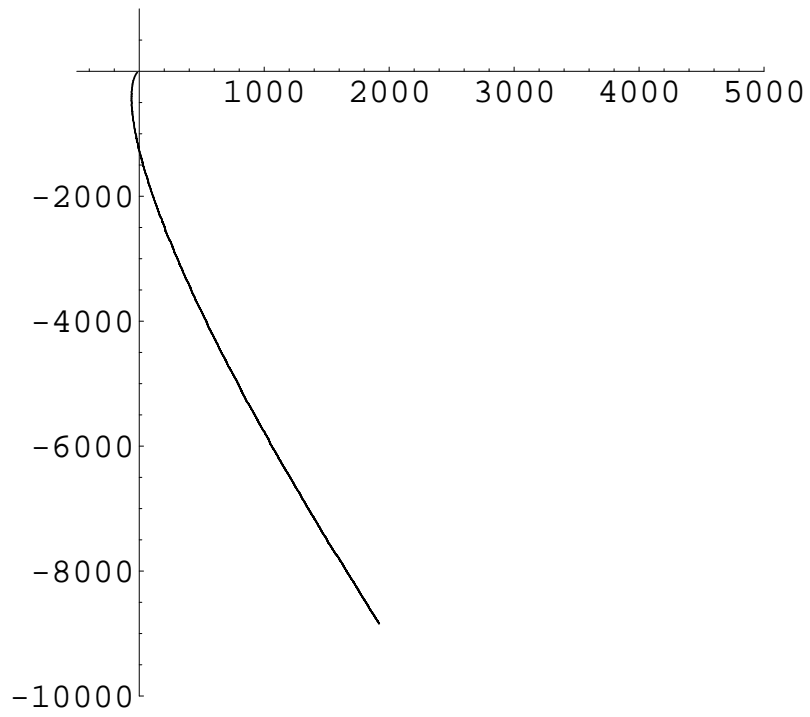

Figure 2:

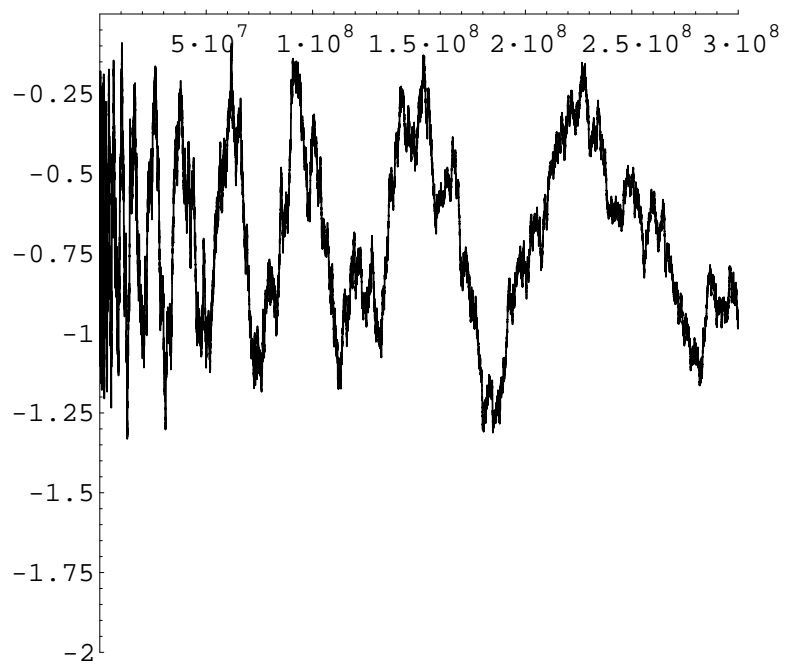


Table 1:

\begin{tabular}{|c|c|c|c|}
$n\left(\times 10^{7}\right)$ & $\frac{1}{\sqrt[3]{x}} \sum_{n \leq x} \mu_{3}(n)$ & $n\left(\times 10^{7}\right)$ & $\frac{1}{\sqrt[3]{x}} \sum_{n \leq x} \mu_{3}(n)$ \\
\hline 1 & $-5.6210-1235.5215 i$ & 16 & $1103.9154-6144.6615 i$ \\
2 & $85.6279-1846.3794 i$ & 17 & $1165.7678-6362.9139 i$ \\
3 & $172.1854-2334.9107 i$ & 18 & $1230.5480-6576.5930 i$ \\
4 & $254.8574-2752.9714 i$ & 19 & $1291.5799-6785.4313 i$ \\
5 & $334.6198-3134.9381 i$ & 20 & $1349.3514-6990.6826 i$ \\
6 & $411.6768-3481.0532 i$ & 21 & $1409.9050-7187.6113 i$ \\
7 & $489.1593-3809.0565 i$ & 22 & $1466.2162-7384.5319 i$ \\
8 & $559.2476-4115.3894 i$ & 23 & $1525.3580-7579.0326 i$ \\
9 & $632.8016-4401.6940 i$ & 24 & $1588.7635-7768.3007 i$ \\
10 & $703.6815-4680.1121 i$ & 25 & $1644.7499-7952.1305 i$ \\
11 & $777.5754-4945.3833 i$ & 26 & $1700.4838-8135.4568 i$ \\
12 & $839.3834-5201.3469 i$ & 27 & $1756.7184-8316.9325 i$ \\
13 & $910.2625-5448.3369 i$ & 28 & $1812.3899-8494.9559 i$ \\
14 & $973.7787-5683.9184 i$ & 29 & $1865.6500-8667.6974 i$ \\
15 & $1039.1155-5914.1483 i$ & 30 & $1920.8040-8841.0852 i$
\end{tabular}

Table 2:

\begin{tabular}{|c|c|c|l|}
$n\left(\times 10^{7}\right)$ & $\frac{1}{\sqrt{x}} \sum_{n \leq x} \mu_{2}(n)$ & $n\left(\times 10^{7}\right)$ & $\frac{1}{\sqrt{x}} \sum_{n \leq x} \mu_{2}(n)$ \\
\hline 1 & -0.266264 & 16 & -0.569684 \\
2 & -1.00847 & 17 & -0.820346 \\
3 & -1.02789 & 18 & -1.26785 \\
4 & -0.5047 & 19 & -1.12899 \\
5 & -1.07593 & 20 & -0.786727 \\
6 & -0.389364 & 21 & -0.669226 \\
7 & -0.788612 & 22 & -0.331841 \\
8 & -0.797382 & 23 & -0.401299 \\
9 & -0.229371 & 24 & -0.61929 \\
10 & -0.3884 & 25 & -0.504067 \\
11 & -0.968909 & 26 & -0.597103 \\
12 & -0.784339 & 27 & -0.8979 \\
13 & -0.978972 & 28 & -1.031 \\
14 & -0.476329 & 29 & -0.95987 \\
15 & -0.264871 & 30 & -0.961173
\end{tabular}


Added in proof. In the meantime, the author proved $\sum_{n \leq x} \mu_{3}(n)=o(x)$, and found a more precise asymptotic formula

$$
\sum_{n \leq x} \mu_{3}(n) \sim \Gamma(\rho)^{-1} \eta_{0} \cdot x(\log x)^{\rho-1}
$$

plausible.

\title{
REFERENCES
}

[1] H. M. Edwards, Riemann's zeta function, Academic Press, 1974.

[2] S. Lang, Algebraic number theory, Addison Wesley, 1970.

\author{
Tomio Kubota \\ Department of Mathematics \\ Meijo University \\ Shiogamaguchi 1-501 \\ Tempaku-ku \\ Nagoya, 468-8502 \\ Japan \\ Mariko Yoshida \\ Department of Mathematics \\ Meijo University \\ Shiogamaguchi 1-501 \\ Tempaku-ku \\ Nagoya, 468-8502 \\ Japan
}

